\title{
Chinese herbal medicine granules (PTQX) for children with moderate to severe atopic eczema: study protocol for a randomised controlled trial
}

\author{
Sherman X. Gu', Anthony L. Zhang ${ }^{1}$, Meaghan E. Coyle ${ }^{1}$, Xiumei Mo², George B. Lenon', Noel E. Cranswick ${ }^{3}$,
} DaCan Chen ${ }^{2,4}$ and Charlie C. Xue $e^{1,4^{*}}$

\begin{abstract}
Background: Atopic eczema or atopic dermatitis is a chronic inflammatory skin disease. Current conventional medical treatment for moderate and severe atopic eczema is not satisfactory. There is promising evidence derived from randomised clinical trials to support the clinical use of Chinese herbal medicine in the management of atopic eczema. However, the available evidence is compromised by the high risk of bias associated with most of the included trials. Therefore, well-designed and adequately powered randomised clinical trials are needed. The primary aim of this trial is to evaluate the efficacy and safety of oral ingestion of an oral Chinese herbal formula (Pei Tu Qing Xin granules; PTQX) in children aged between 6 and 16 years with moderate to severe atopic eczema.

Methods/Design: We have designed a randomised, double-blind, placebo-controlled, two-arm, parallel clinical trial with 12 weeks of treatment and a 4-week follow-up period. A pilot study with 30 participants will be conducted at the RMIT University in Australia to determine the feasibility of the full-scale randomised clinical trial $(N=124)$. Eczema Area and Severity Index score will be the primary outcome. Secondary outcome measures include change in symptoms using the Patient-Oriented Eczema Measure, the Children's Dermatology Life Quality Index and the use of concomitant medicines. Safety parameters include report of adverse events and pathology tests during the trial period.
\end{abstract}

Discussion: Key elements for conducting a high-quality randomised clinical trial have been addressed in this protocol. Findings from the proposed trial will provide critical evidence regarding Chinese herbal medicine treatment for atopic eczema.

Trial registration: Australian New Zealand Clinical Trials Registry Identifier: ACTRN12614001172695. Date of Registration: 7 November 2014.

Keywords: Atopic dermatitis, Atopic eczema, Chinese herbal medicine, Clinical trial, PTQX, RCT

\section{Background}

Atopic eczema (AE) or atopic dermatitis is an inflammatory skin disease characterised by redness of the skin, scaling, swelling, accentuation of the hair follicles and lichenification that result from chronic scratching owing

\footnotetext{
* Correspondence: charlie.xue@rmit.edu.au

'Traditional and Complementary Medicine Research Program, Health Innovations Research Institute and School of Health Sciences, Royal Melbourne Institute of Technology (RMIT) University, Plenty Road, P.O. Box 71, Bundoora 3083, VIC, Australia

${ }^{4}$ The Guangdong Provincial Academy of Chinese Medical Sciences, 111 Dade Road, Guangzhou 510120, Guangdong, China

Full list of author information is available at the end of the article
}

to severe itchiness [1]. The prevalence of $\mathrm{AE}$ worldwide ranges from $1 \%$ to $20 \%$. Nigeria, the United Kingdom and New Zealand have the highest reported prevalences [2]. A recent Australian prospective cohort study done over the course of 40 years showed that childhood eczema was strongly associated with the incidence and persistence of adult atopic asthma, suggesting that early treatment may reduce morbidity later in life [3].

Management of mild AE includes topical application of corticosteroids and emollients, and refractory moderate or severe $\mathrm{AE}$ is managed with systemic therapies, including oral steroids or immunosuppressive drugs

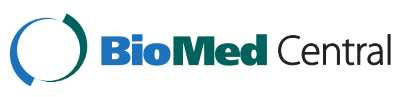

(C) 2015 Gu et al. This is an Open Access article distributed under the terms of the Creative Commons Attribution License (http://creativecommons.org/licenses/by/4.0), which permits unrestricted use, distribution, and reproduction in any medium, provided the original work is properly credited. The Creative Commons Public Domain Dedication waiver (http:// creativecommons.org/publicdomain/zero/1.0/) applies to the data made available in this article, unless otherwise stated. 
such as azathioprine, cyclosporine, mycophenolate and methotrexate $[4,5]$. Current conventional medical treatment for moderate and severe $\mathrm{AE}$ is not satisfactory, as long-term oral corticosteroid use can lead to adverse reactions such as suppression of the hypothalamic-pituitary-adrenal axis, osteoporosis, aseptic necrosis of the hip, hypertension, ocular changes and altered immune function [6]. Side effects of immunosuppressive medications, in particular the risk of hepatosplenic T-cell lymphomas associated with azathioprine, have raised alert [7]. Therefore, many people with AE may choose to use Chinese herbal medicine $(\mathrm{CHM})$ for the treatment of $\mathrm{AE}[8]$.

CHM has a long history of use, dating back to the second century AD. Unlike conventional medicine, CHM uses a composite of different botanical resources such as barks, seeds, flowers, roots or mineral substances to create a formula which is administered as decoctions, pills, washes, lotions or ointments. There has been increasing interest in evaluating the efficacy and safety of $\mathrm{CHM}$ for $\mathrm{AE}$. To determine the benefit of $\mathrm{CHM}$ for $\mathrm{AE}$, we completed a Cochrane systematic review of $\mathrm{CHM}$ for $\mathrm{AE}$ in 2013 [9]. A total of 28 studies were included in the review (2306 participants). There is evidence that CHM has increasingly been used for the management of $\mathrm{AE}$ since the publication of the first Cochrane systematic review in this area in 2004. All included trials have presented the benefit of CHM was superior or equivalent to its comparator. However, the promising effect claimed by the trials' investigators is compromised by the high risk of bias within most of the included trials, and we further suggested that well-designed, adequately powered randomised clinical trials (RCTs) are needed.

\section{Trial objective}

The primary objective of the present trial is to determine the efficacy and safety of oral ingestion of an existing oral Chinese herbal formula (Pei Tu Qing Xin granules; PTQX) on symptom severity and improvement in health-related quality of life (QoL) in children aged between 6 and 16 years with moderate to severe AE. We will also assess the safety of PTQX for AE.

\section{Methods/Design}

The trial will be a 16-week, randomised, double-blind, placebo-controlled, two-arm, parallel clinical trial. The 16-week trial includes 12 weeks of treatment with either PTQX or placebo and a 4-week follow-up period. Figure 1 outlines the process of the trial. A pilot study $(N=30)$ will be conducted to inform a planned larger RCT $(N=124)$. The study protocol was approved by the Royal Melbourne Institute of Technology (RMIT) University Human Research Ethics Committee (HREC), filed with the Therapeutic Goods Administration (TGA) under the Clinical Trial Notification (CTN) scheme (CTN file number: 2014/033590) and registered with the Australian and New Zealand Clinical Trials Registry (ANZCTR) (registration ID: ACTRN12614001172695; dated 7 November 2014).

\section{Randomisation}

A block randomisation method will be employed with balanced variable blocks. Equal numbers of participants will receive the real treatment and the inert vehicle (the placebo). Randomisation codes will be generated by a computer program run by an independent statistician who is not directly involved with the trial.

\section{Blinding and allocation concealment}

Neither the trial participants nor investigators will know the participant allocation to receive real treatment or placebo. The randomisation codes will be concealed in individual opaque envelopes. Envelopes will be sequentially numbered and opened only after the participant's unique identifier is written on the envelope. At the time of randomization (visit 1 , week 0 ), each participant will randomly pick one sealed envelope from among the group. The envelope will be opened by a CHM dispenser, who will be a registered CHM practitioner or registered CHM dispenser. The trial medication (both PTQX and placebo) will be prepackaged, and the appearance of the packages will be identical. The randomisation code will not be revealed to participants and personnel who are involved in data entry and data analysis. An emergency unblinding envelope with identical contents will be prepared in case a serious adverse event occurs during the treatment period. In case of a serious adverse event where immediate cessation of trial medication is required, the emergency unblinding envelope will be opened for further investigation of the nature of the event by the trial investigators.

\section{Preparation and administration of the trial medications}

The trial medications will be prepared by Jiangyin Tianjiang Pharmaceutical Co. Ltd, a Good Manufacturing Practicescertified CHM extract granules manufacturer. Quality control and quality assurance, such as identification of products and quality and safety testing, were conducted by the manufacturer. The raw herbs were mixed, cooked twice with water, filtered, concentrated, spray-dried and mixed with sucralose and pearl powder (Margarita powder) and finally forming granules (PTQX). They were packaged in small sachets weighing $2 \mathrm{~g}$ each. A sample batch of PTQX was tested for microorganisms and is deposited with the manufacturer for traceability. In addition, a fingerprint profile of PTQX has been established by using an ultra high-performance liquid chromatographyhigh resolution mass spectrometry operated in multistage 


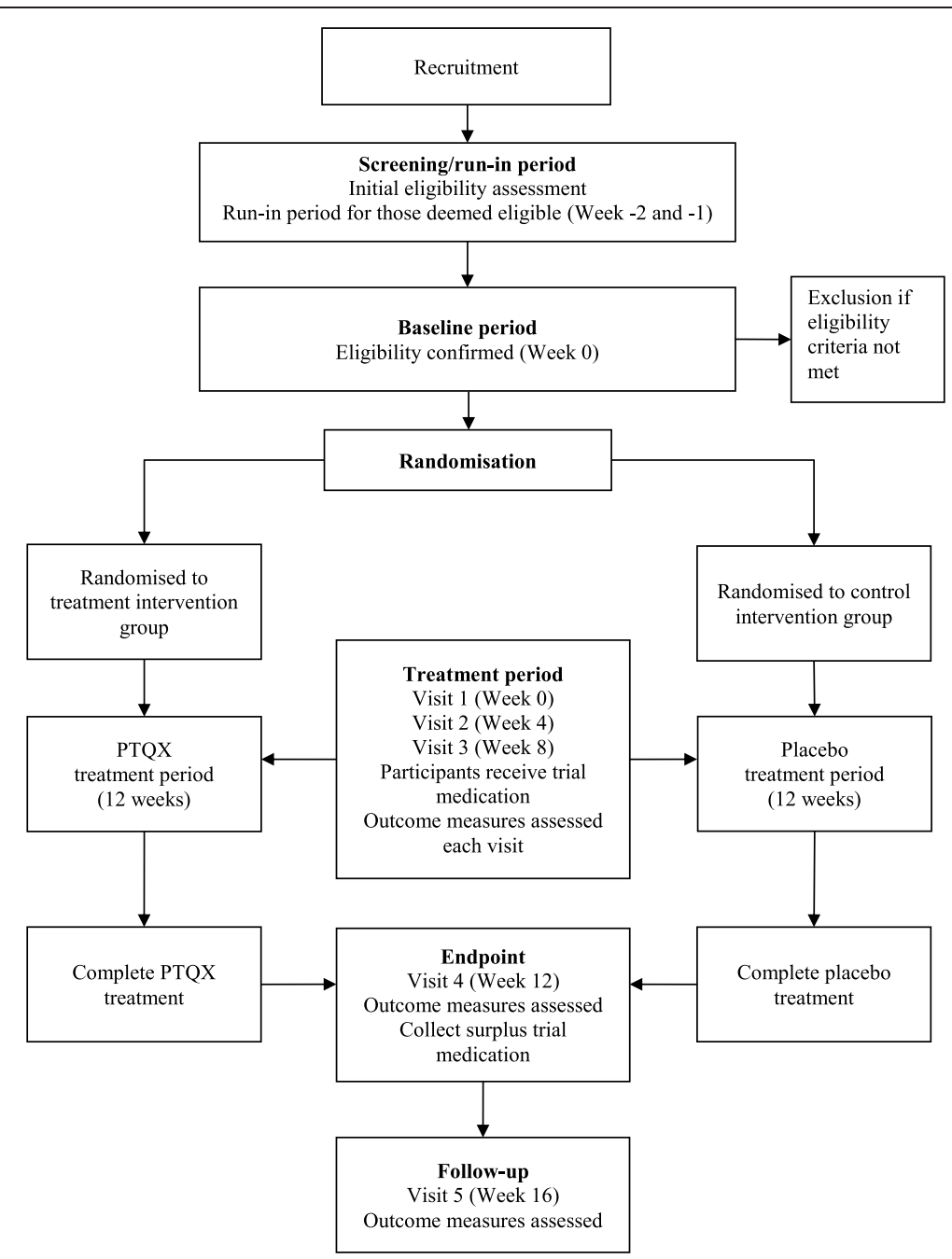

Fig. 1 Flowchart of the trial process

mode profiling method for further study of the active compounds of the formula.

PTQX is modified from a CHM formula, Qing Xin Pei $\mathrm{Tu}$, which is based on the principle of treatment in strengthening the spleen and eliminating the heart fire in Chinese medicine, and most children with AE manifested with deficiency of the spleen and heart fire $[10,11]$. The formula has been used in children with AE for many years [10-12]. The formula is prepared as CHM granules with extracts from nine Chinese medicinal substances, including Rhizoma Atractylodis macrocephalae (Bai Zhu), Radix Pseudostellariae (Tai Zi Shen), Rhizoma Dioscoreae (Shan Yao), Semen Coicis (Yi Yi Ren), Rhizoma Imperatae (Bai Mao Gen), Fructus Forsythiae (Lian Qiao), Radix Glycyrrhizae (Gan Cao), Cortex Dictamni (Bai Xian Pi) and Margarita (Zhen Zhu Feng). In CHM theory, Bai Zhu has the function of strengthening the spleen, and Lian Qiao can clear away the heart fire and remove toxins.
These two herbs serve as sovereign (jun) herbs. Bai Mao Gen is used to cool the blood and reduce swelling by promoting diuresis. Tai Zi Shen, Yi Yi Ren and Shan Yao have the function of strengthening the spleen and eliminating dampness. These four substances act as minister (chen) herbs to further enhance the effects of the sovereign herbs. Zhen Zhu Feng calms the mind and extinguishes the wind. Bai Xian Pi has the function of clearing away heat, drying the dampness, expelling the wind and easing itchiness. These two ingredients work as assistant (zuo) role in the formula. Can Cao is a guide (shi) herb, as it harmonises all ingredients of the formula [13]. Evidence derived from preclinical research have shown that Atractylodis macrocephalae and Semen Coicis could improve the immune function by increasing interleukin (IL)-1 or IL-2, and Fructus Forsythiae and Radix Glycyrrhizae have anti-inflammatory action [14]. 
Four grams of trial medications twice daily (total of $8 \mathrm{~g}$ ) for 6-7-year-old subjects and $6 \mathrm{~g}$ twice daily (total of $12 \mathrm{~g}$ ) for 8-16-year-old participants will be given to both study groups with written and oral instructions on use of the trial medications.

\section{Selection and withdrawal of participants}

Participants will be recruited through advertisements in local papers, school newsletters, posters, via the internet and through referrals from general practitioners (GPs) or dermatologists. Trial information flyers will be placed in offices of GPs or dermatologists who agree to refer patients to the trial. Contact details of the potential participants who express interest in the trial will be recorded. The participant information and consent form with information about the trial, including the purpose of the trial, details of the intervention, and potential adverse reactions, will be given to potential participants. Potential participants will be interviewed to assess their eligibility against the inclusion and exclusion criteria to select suitable participants for the trial (screening and run-in period).

\section{Selection criteria}

The U.K. Working Party's diagnostic criteria and the Nottingham Eczema Severity Score (NESS) will be adopted as inclusion criteria for this trial [15-17]. Eligible participants will be boys or girls aged 6-16 years with a history of skin pruritus within the previous 12 months plus three or more of the following: (1) onset under the age of 2 years, (2) history of flexural involvement, (3) history of generally dry skin, (4) personal history of other atopic disease such as asthma or (5) visible flexural dermatitis. Further, eligible participants will have a total NESS score $\geq 9$ (moderate to severe) at baseline and will have provided written informed consent.

Those who used corticosteroids, other immunosuppressives or any preparation of oral herbal medicines for treatment of $\mathrm{AE}$ in the past 30 days or who have been diagnosed with scabies, allergic contact dermatitis, seborrhoeic dermatitis or psoriasis will be excluded. Those who have severe medical conditions, such as cardiovascular, liver or renal dysfunction, and/or a history of lactose intolerance will not be included.

\section{Withdrawal, dropout and discontinuation}

Participants are free to withdraw at any time during the trial. Participants who wish to withdraw will be offered the option to cease trial medication but continue attending scheduled visits for outcome measurement. Participants who withdraw will be followed to investigate the reason for withdrawal. Participants may be advised to discontinue the treatment if there is a product-related adverse event of a serious nature or if the participant was not compliant with the study requirements (e.g., use of oral corticosteroids during the treatment period). Topical corticosteroid use is allowed for those participants who were using topical corticosteroids before trial participation and whose skin conditions worsen during the trial treatment period. These concomitant medications will be documented. Discontinuers will not be replaced by new participants. Intention-to-treat analysis will be performed on missing data from discontinuers with the last observation carried forward method.

\section{Sample size}

Calculation of the sample size of this trial was based on the effect size of the primary outcome, Eczema Area and Severity Index (EASI) score, reported in a previous published study [18]. The means and standard deviations (SDs) of the EASI scores were $7.4 \pm 3.3$ and $9.6 \pm 4.1$ for the active treatment and control groups, respectively. To achieve $90 \%$ power $(\beta=0.10)$, significance level of 0.05 $(\alpha)$ in a two-tailed test, calculated by using $G^{*}$ Power 3.1.2 (http://www.gpower.hhu.de/en.html), 62 participants, based on an estimated $10 \%$ dropout rate, are needed for each group. That makes a total of 124 for the main trial. A pilot study with 30 participants (15 per group) conducted in RMIT University will provide preliminary data on the clinical efficacy of PTQX based on EASI score and will allow us to monitor for adverse events. The mean and SD of EASI score from the pilot study will be used for recalculation of the sample size for the main trial.

\section{Screening and run-in, baseline, treatment periods and endpoint}

Preliminary screening assessments will be conducted during the 2 weeks of the run-in period (weeks -2 and -1 ). Only those participants who have met the inclusion and exclusion criteria will be randomised. EASI score and other baseline outcome assessments will be measured.

The treatment period will involve oral ingestion of the trial medications twice daily for 12 weeks. The trial medications will be supplied to participants at visit 1 (week 0 ), visit 2 (week 4) and visit 3 (week 8 ). The endpoint is set at visit 4 (week 12) of the trial period.

\section{Outcome measures}

Current recommendations for outcome measurement in RCTs of AE include clinical signs and symptoms, longterm control of flares and health-related QoL [19]. This trial will adopt these recommendations for its outcome measures. The primary outcome measure is change in clinical signs severity based on EASI score. Secondary outcome measures include change in symptoms using the Patient-Oriented Eczema Measure (POEM) [20], health-related QoL using the Children's Dermatology 
Life Quality Index (CDLQI) [21], use of concomitant medicines (number of days used and total dosage for corticosteroids) and adverse events and safety (pathology tests).

The EASI, POEM, CDLQI, concomitant medications and adverse events will be recorded at visit 1 (week 0 ), visit 2 (week 4), visit 3 (week 8), visit 4 (week 12) and visit 5 (week 16). Pathology tests (full blood examination with eosinophil count and immunoglobulin E, liver and renal function tests) will be performed by a qualified phlebotomist at the screening (week -2) and at the second (week 4) and fourth (week 12) visits. Medical advice from a medical officer will be sought for any serious adverse events or for any serious aggravation of the existing skin condition or abnormal pathology results during the trial period.

Any adverse events will be managed in compliance with the guidelines of the Note for Guidance on Clinical Safety Data Management: Definitions and Standards for Expedited Reporting (CPMP/ICH/377/95) issued by TGA [22]. A serious adverse event will be reported to the RMIT HREC within 24 hours and then to the TGA within 7-15 days. Table 1 summarises the schedule of the trial and outlines the time points of outcome measures of the trial.

\section{Statistical analysis}

Data will be analysed by an independent statistician using IBM SPSS version 22 software (IBM, Armonk, NY, USA). Comparison of means of continuous variables (i.e., EASI, POEM and CDLQI scores) will be expressed as differences in means with $95 \%$ confidence intervals (CIs). One-way analysis of variance will be used for normally distributed data, and nonparametric tests will be used for skewed distribution data. $\chi^{2}$ or Fisher's exact tests will be performed for comparison of dichotomised data, and dichotomous data will be expressed as risk ratios with $95 \%$ CIs. All comparisons will be twotailed, with $p$ values $<0.05$ considered to be statistically significant.

\section{Discussion}

Determination of the trial timeline is one of the challenges of the trial design. It depends on many factors, such as how long the effect of the intervention may last, costs of the trial and tolerability by and compliance of the participants. The trial duration of 16 weeks has been determined after consideration of these issues. This protocol was developed according to the guidelines of Australian Clinical Trial Handbook [23] and follows the nine CONSORT checklist items for RCTs of herbal medicines [24]. Key elements for conducting a high-quality $\mathrm{RCT}$, such as randomisation, sequence generation, allocation concealment, blinding methods, proper sample size, compliance with protocol, determination of baseline and endpoint and adequate outcome measures have been addressed.

\section{Trial status}

Participant recruitment commenced on 31 March 2015. It is anticipated that the trial will be completed in February 2016.

Table 1 Schedule of the trial

\begin{tabular}{|c|c|c|c|c|c|c|}
\hline Items & Screening/run-in period & Visit 1 (baseline) & Visit 2 & Visit 3 & Visit 4 (endpoint) & Visit 5 (follow-up) \\
\hline Week of the trial & $-2 /-1$ & 0 & 4 & 8 & 12 & 16 \\
\hline Demographic information-taking & + & & & & & \\
\hline Informed consent form (PICF) & + & & & & & \\
\hline General medical history-taking & + & & & & & \\
\hline $\begin{array}{l}\text { Severity of AE for inclusion and exclusion of } \\
\text { the participants (NESS) }\end{array}$ & + & & & & & \\
\hline Blood tests & + & & + & & + & \\
\hline Clinical sign of $\mathrm{AE}$ (EASI) & & + & + & + & + & + \\
\hline Symptoms (POEM) & & + & + & + & + & + \\
\hline QoL score (CDLQI) & & + & + & + & + & + \\
\hline Distributions of treatment or placebo products & & + & + & + & & \\
\hline Daily medical record & + & + & + & + & + & + \\
\hline Monitor of adverse event & & + & + & + & + & + \\
\hline Compliance check & & + & + & + & + & + \\
\hline Survey of participation & & & & & & + \\
\hline
\end{tabular}

Abbreviations: AE atopic eczema, CDLQ/ Children's Dermatology Life Quality Index, EASI Eczema Area and Severity Index, NESS Nottingham Eczema Severity Score, PICF participant information and consent form, POEM Patient-Oriented Eczema Measure, QoL quality of life 


\section{Abbreviations}

AE: Atopic eczema; ANZCTR: Australian New Zealand Clinical Trials Registry; CDLQI: Children's Dermatology Life Quality Index; CHM: Chinese herbal medicine; Cl: Confidence interval; CONSORT: Consolidated Standards of Reporting Trials; CTN: Clinical Trial Notification; EASI: Eczema Area and Severity Index; GP: General practitioner; HREC: Human Research Ethics Committee; IL: Interleukin; NESS: Nottingham Eczema Severity Score; PICF: Participant information and consent form; POEM: Patient-Oriented Eczema Measure; PTQX: Pei Tu Qing Xin granules; QoL: Quality of life; RCT: Randomised clinical trial; RMIT: Royal Melbourne Institute of Technology; SD: Standard deviation; TGA: Therapeutic Goods Administration.

\section{Competing interests}

DC developed the CHM formula, which has been patented. All other authors declare that they have no competing interests.

\section{Authors' contributions}

SXG, CCX, ALZ, DC and MEC designed and developed the trial protocol. SXG drafted the trial protocol. GBL, XM, DC and NEC provided expert advice and critical comments on the protocol. All authors read and approved the final manuscript.

\section{Authors' information}

SXG: BMed, MAppSc, PhD candidate, RMIT University

ALZ: BMed, MPH, PhD, Head, Discipline of Chinese Medicine, School of the Health Sciences, RMIT University

MEC: BlthSc(Acup), PhD, Research Fellow, Discipline of Chinese Medicine, School of the Health Sciences, RMIT University

XM: BMed, MMed, PhD, Dermatologist, Guangdong Provincial Hospital of Chinese Medicine

GBL: BHlthSc (Hons), PhD, Lecturer, Discipline of Chinese Medicine, School of the Health Sciences, RMIT University

NEC: MBBS, BMedSc, LLB, FRACP, Paediatrician and Clinical Pharmacologist,

Royal Children's Hospital, Melbourne

DC: BMed, MMed, Dermatologist, Guangdong Provincial Hospital of Chinese Medicine; Principal, Guangdong Provincial Academy of Chinese Medical Sciences

CCX: BMed, PhD, Head, School of the Health Sciences, RMIT University; Senior Principal Research Fellow, Guangdong Provincial Academy of Chinese Medical Sciences

\section{Acknowledgements}

The authors thank Associate Professor Clifford Da Costa for his advice on sample size calculation. The authors also thank the Jiangyin Tianjiang Pharmaceutical Co. Ltd., the manufacturer of PTQX, for providing the trial medications. The company will not be involved in any other aspect of trial design or implementation or in the analysis or publication of the results. This project is funded by the China-Australia International Research Centre for Chinese Medicine and the School of Health Sciences, RMIT University. The trial is sponsored by RMIT University and Guangdong Provincial Academy of Chinese Medical Sciences, China.

\section{Author details \\ ${ }^{1}$ Traditional and Complementary Medicine Research Program, Health Innovations Research Institute and School of Health Sciences, Royal Melbourne Institute of Technology (RMIT) University, Plenty Road, P.O. Box 71, Bundoora 3083, VIC, Australia. ${ }^{2}$ Department of Dermatology, Guangdong Provincial Hospital of Chinese Medicine, 111 Dade Road, Guangzhou 510120, Guangdong, China. ${ }^{3}$ Royal Children's Hospital, Murdoch Children's Research Institute, University of Melbourne, 50 Flemington Road, Parkville 3052, VIC, Australia. ${ }^{4}$ The Guangdong Provincial Academy of Chinese Medical Sciences, 111 Dade Road, Guangzhou 510120, Guangdong, China.}

\section{Received: 18 February 2015 Accepted: 10 June 2015 Published online: 07 July 2015}

\section{References}

1. Williams H, Bigby M, Diepgen T, Herxheimer A, Naldi L, Rzany B, editors. Evidence-based dermatology. 2nd ed. Oxford: Blackwell; 2008.

2. DaVeiga SP. Epidemiology of atopic dermatitis: a review. Allergy Asthma Proc. 2012;33(3):227-34.
3. Martin PE, Matheson MC, Gurrin L, Burgess JA, Osborne N, Lowe AJ, et al. Childhood eczema and rhinitis predict atopic but not nonatopic adult asthma: a prospective cohort study over 4 decades. J Allergy Clin Immunol. 2011;127(6):1473-9.

4. Tan AU, Gonzalez ME. Management of severe atopic dermatitis in children. J Drugs Dermatol. 2012;11(10):1158-65.

5. Hon $\mathrm{KL}$, Chan $\mathrm{BC}$, Leung $\mathrm{PC}$. Chinese herbal medicine research in eczema treatment. Chin Med. 2011;6:17.

6. Walling HW, Swick BL. Update on the management of chronic eczema: new approaches and emerging treatment options. Clin Cosmet Investig Dermatol. 2010;3:99-117.

7. Denby KS, Beck LA. Update on systemic therapies for atopic dermatitis. Curr Opin Allergy Clin Immunol. 2012;12(4):421-6.

8. Hon KL, Ma KC, Wong Y, Leung TF, Fok TF. A survey of traditional Chinese medicine use in children with atopic dermatitis attending a paediatric dermatology clinic. J Dermatolog Treat. 2005;16(3):154-7.

9. Gu S, Yang AW, Xue CC, Li CG, Pang C, Zhang W, et al. Chinese herbal medicine for atopic eczema. Cochrane Database Syst Rev. 2013;9, CD008642.

10. Huang YJ, Liu JF, Chen DC. [Professor DaCan Chen's clinical experience in treatment of atopic dermatitis]. Chin J Dermatovenerol Integr Tradit West Med. 2010;9(3):165-6. Chinese.

11. Sun XD. [Professor DaCan Chen's experience in treatment of atopic dermatitis by the heart and spleen theory]. Chin J Dermatovenerol Integr Tradit West Med. 2006;5(1):55-6. Chinese.

12. Chen DC, Liu C. Theory and practice of atopic dermatitis in its constitution, etiology and pathogenesis of the heart and spleen. J New Chin Med. 2009;41(8):7-8

13. Chen D, inventor; Google Patents, assignee. Chinese medicinal composition for treating atopic dermatitis and preparation method thereof. Publication CN 103393981 A. Application CN 201310328668. 20 November 2013. http://www.google.com/patents/CN103393981A?cl=en. Accessed 22 May 2015.

14. Shen YJ. Pharmacology of Chinese medicines. Beijing: People's Medical Publishing House; 2000. p. 51-2. 81-83, 190-3, 208-18. Chinese.

15. Charman C, Chambers C, Williams H. Measuring atopic dermatitis severity in randomized controlled clinical trials: what exactly are we measuring? J Investig Dermatol. 2003;120(6):932-41.

16. Williams HC, Burney PG, Hay RJ, Archer CB, Shipley MJ, Hunter JJ, et al. The U.K. Working Party's Diagnostic Criteria for Atopic Dermatitis. I. Derivation of a minimum set of discriminators for atopic dermatitis. Br J Dermatol. 1994;131(3):383-96.

17. Emerson RM, Charman CR, Williams HC. The Nottingham Eczema Severity Score: preliminary refinement of the Rajka and Langeland grading. Br J Dermatol. 2000;142(2):288-97.

18. Ma YB, Sun LY, Wang P, Zhang GZ, Cai NN. Clinical observation of combination of Jianpi Runfu decoction and topical application of Gancao oil for atopic dermatitis with syndrome of spleen deficiency and blood dryness. Beijing J Tradit Chin Med. 2010;29(9):680-2.

19. Schmitt J, Langan S, Stamm T, Williams HC, Harmonizing Outcome Measurements in Eczema (HOME) Delphi panel. Core outcome domains for controlled trials and clinical recordkeeping in eczema: international multiperspective Delphi consensus process. J Invest Dermatol. 2011;131(3):623-30.

20. Charman CR, Venn AJ, Williams HC. The Patient-Oriented Eczema Measure: development and initial validation of a new tool for measuring atopic eczema severity from the patients' perspective. Arch Dermatol. 2004;140(12):1513-9.

21. Lewis-Jones MS, Finlay AY. The Children's Dermatology Life Quality Index (CDLQI): initial validation and practical use. Br J Dermatol. 1995;132(6):942-9.

22. Therapeutic Goods Administration (TGA), Commonwealth Department of Health and Aged Care. Note for guidance on clinical safety data management: definitions and standards for expedited reporting (CPMP/ICH/ 377/95). Canberra, Australia: TGA; July 2000. https://www.tga.gov.au/sites/ default/files/ich37795.pdf. Accessed 18 June 2015.

23. Therapeutic Goods Administration (TGA), Department of Health and Ageing, Australian Government. The Australian clinical trial handbook: a simple, practical guide to the conduct of clinical trials to international standards of Good Clinical Practice (GCP) in the Australian context. Canberra: Commonwealth of Australia; March 2006. https://www.tga.gov.au/sites/ default/files/clinical-trials-handbook.pdf. Accessed 18 June 2015.

24. Gagnier JJ, Boon H, Rochon P, Moher D, Barnes J, Bombardier C. Reporting randomized, controlled trials of herbal interventions: an elaborated CONSORT statement. Ann Intern Med. 2006;144(5):364-7. 